\title{
Impacto da terapia Reiki no bem-estar dos pacientes oncológicos: Uma revisão
}

\section{sistemática}

\author{
Impact of Reiki therapy on the well-being of oncological patients: A systematic review \\ Impacto de la terapia Reiki en el bienestar de los pacientes oncológicos: Una revisión sistemática
}

Recebido: 30/06/2021 | Revisado: 06/07/2021 | Aceito: 10/07/2021 | Publicado: 22/07/2021

\author{
Vitória Martins Castro Feitosa \\ ORCID: https://orcid.org/0000-0001-6907-9536 \\ Centro Universitário de Patos, Brasil \\ E-mail: vitoriamcfeitosa@gmail.com \\ Yngrid Maria Torres Freire \\ ORCID: https://orcid.org/0000-0001-6858-2834 \\ Centro Universitário de Patos, Brasil \\ E-mail: freireyngrid@hotmail.com \\ Hosana Barros Capuxú \\ ORCID: https://orcid.org/0000-0001-9999-8314 \\ Centro Universitário de Patos, Brasil \\ E-mail: hosanabcapuxu@hotmail.com \\ Raniere Leite Dóia Filho \\ ORCID: https://orcid.org/0000-0001-8367-549X \\ Centro Universitário de Patos, Brasil \\ E-mail: ranifilhoo@gmail.com \\ Milena Nunes Alves de Sousa \\ ORCID: https://orcid.org/0000-0001-8327-9147 \\ Centro Universitário de Patos, Brasil \\ E-mail: milenanunes@fiponline.edu.br
}

\begin{abstract}
Resumo
Objetivo: Analisar em pacientes com câncer, se o uso de Reiki associado ao tratamento convencional, em comparação apenas à terapêutica padrão promove a melhora do bem-estar e/ou quadro clínico. Métodos: Realizou-se uma revisão sistemática da literatura, com busca nas bases de dados da Biblioteca Virtual em Saúde (BVS) e Medical Publisher (PUBMED), seleção dos artigos a partir dos Descritores em Ciências da Saúde (DeCS) combinados: "Therapeutic Touch" AND Neoplasms and Therapeutics AND "Treatment Outcome". A avaliação da qualidade metodológica e qualidade das evidências foi feita a partir do Sistema GRADE. Resultados: Foram selecionados cinco estudos, quatro deles disponíveis na BVS, com nível de evidência 1B e todos publicados nos Estados Unidos. A maioria apontou uma melhora significativa com uso de Reiki associado à terapêutica tradicional quando comparado ao tratamento padrão isolado, e relatou a redução de células natural killers, contendo uma relação com a citotixicidade dessas e aumento significativo na hemoglobina e no hematócrito de algumas pacientes. Ademais, asseveraram que a terapia de toque é um tratamento alternativo que propicia melhora dos sintomas como diminuição da dor, relaxamento, redução de estresse, ansiedade, fadiga, como também aumentou a vivacidade e o humor dos pacientes. Conclusão: Essa terapia complementar pode ser um instrumento favorável ao gerenciamento dos pacientes oncológicos, visto que aumenta a qualidade de vida durante o recurso terapêutico padrão.
\end{abstract}

Palavras-chave: Medicina integrativa; Reiki; Terapêutica; Oncologia integrativa; Qualidade de vida.

\begin{abstract}
Abstract: To analyze in patients with cancer if the use of Reiki associated with conventional treatment, compared to only conventional therapy, promotes an improvement in welfare and/or clinical condition. Methods: A systematic review of the literature was made, with a search in the databases of the Virtual Health Library (VHL) and Medical Publisher (PUBMED), selection of articles from the combined Health Sciences Descriptors (DeCS): "Therapeutic Touch" AND Neoplasms and Therapeutics AND "Treatment Outcome". The evaluation of methodological quality and quality of evidence was made using the GRADE system. Results: Five studies were selected, four of them available in the VHL, with level of evidence 1B and all published in the United States. Most of them showed a significant improvement with the use of Reiki associated with traditional therapy when compared to standard treatment alone, and reported the reduction of natural killer cells, with a relation to their cytotoxicity and a significant increase in hemoglobin and hematocrit in some patients. Furthermore, they affirmed that touch therapy is an alternative treatment that provides improvement of symptoms such as pain reduction, relaxation, stress reduction, anxiety, fatigue, as well as increased
\end{abstract}


vivacity and mood of the patients. Conclusion: This complementary therapy can be a favorable instrument for the management of cancer patients, since it increases quality of life during the standard therapeutic resource.

Keywords: Integrative medicine; Reiki; Therapeutics; Integrative oncology; Quality of life.

\section{Resumen}

Objetivo: Analizar, en pacientes oncológicos, si el uso de Reiki asociado al tratamiento convencional, en comparación con la terapia estándar sola, promueve una mejora del bienestar y / o estado clínico. Métodos: Se realizó una revisión bibliográfica sistemática, con búsqueda en las bases de datos de la Biblioteca Virtual en Salud (BVS) y Medical Publisher (PUBMED), selección de artículos de los Descriptores de Ciencias de la Salud (DeCS) combinados: "Toque Terapéutico" Y Neoplasias y Terapéutica. Y "Resultado del tratamiento". La evaluación de la calidad metodológica y la calidad de la evidencia se realizó mediante el sistema GRADE. Resultados: Se seleccionaron cinco estudios, cuatro de ellos disponibles en la BVS, con nivel de evidencia 1B y todos publicados en Estados Unidos. La mayoría mostró una mejora significativa con el uso de Reiki asociado con la terapia tradicional en comparación con el tratamiento estándar solo, y reportaron una reducción de las células asesinas naturales, con una relación con su citotoxicidad y un aumento significativo de la hemoglobina y el hematocrito en algunos pacientes. Además, afirmaron que la terapia táctil es un tratamiento alternativo que mejora síntomas como disminución del dolor, relajación, reducción del estrés, ansiedad, fatiga, así como aumento del estado de alerta y estado de ánimo de los pacientes. Conclusión: esta terapia complementaria puede ser un instrumento favorable para el manejo del paciente oncológico, ya que aumenta la calidad de vida durante el recurso terapéutico estándar.

Palabras clave: Medicina integrativa; Reiki; Terapia; Oncología integrativa; Calidad de vida.

\section{Introdução}

O câncer compreende mais de 100 doenças e tem aumentado sua incidência nos últimos anos, seja pelo aumento da exposição a fatores cancerígenos, envelhecimento da população ou melhora das tecnologias de diagnóstico. Por definição, é o crescimento desordenado de células com capacidade de disseminação entre tecidos e órgãos adjacentes à região afetada inicialmente (Batista; Mattos; Silva, 2015).

Para os autores citados, o tratamento oncológico quimioterápico, o mais comum dentre os tratamentos para as neoplasias, é agressivo e pode causar sofrimento, como dor física e fragilidade. Além disso, existem efeitos adversos inerentes a ele, que incluem náuseas, vômitos, perda de peso, alopecia, fadiga, ansiedade, dispneia, depressão e astenia.

Nessa perspectiva, o processo do câncer, desde o diagnóstico até o tratamento, é muito doloroso ao paciente (Alarcão; Fonseca, 2016). Causa problemas físicos, psíquicos e sociais pelas manifestações clínicas e estigmatização da doença, o que dificulta a adesão à terapêutica e reduz consideravelmente a qualidade de vida do paciente (Batista; Mattos; Silva, 2015; Nascimento; Sousa; Alencar, 2020).

Na literatura, existem evidências dos benefícios da terapia Reiki no tratamento do câncer (Kirshbaum; Stead; Bartys, 2016). Uma terapia surgida na cultura japonesa, na qual há a imposição das mãos para canalizar energia vital, com o intuito de melhorar a resposta natural do corpo e auxiliar na recuperação e manutenção da saúde. A partir de 2017, essa prática passou a ser integrada ao Sistema Único de Saúde (SUS) e ofertada à população geral de acordo com a ampliação da Política Nacional de Práticas Integrativas e Complementares em Saúde (PNPIC). Seu significado é plural, complexo e subjetivo, pois compreende um olhar multidimensional do ser humano, que vai além do campo biológico (Amarello; Castellanos; Souza, 2021) (Brasil, 2015).

Para os autores, seu uso é baseado na melhora da dor, do estresse e da ansiedade, de modo a aumentar a qualidade de vida, amplificar a força de enfrentamento à doença e proporcionar o bem-estar. Diante disso, este artigo teve por objetivo de analisar em pacientes com câncer, se o uso de Reiki associado ao tratamento convencional, em comparação apenas à terapêutica padrão promove a melhora do bem-estar e/ou quadro clínico.

O estudo justifica-se pelo fato de o objeto de estudo ainda ser pouco elucidado, portanto, pode ser de extrema importância como auxílio aos profissionais ao decidirem qual conduta será seguida, visto que possivelmente seria uma excelente via alternativa para minimizar o sofrimento dos pacientes. 


\section{Metodologia}

A revisão sistemática da literatura é uma pesquisa científica que segue protocolos específicos, detalhando explicitamente cada passo na construção do estudo, as limitações de cada artigo e da própria revisão. Ela busca analisar trabalhos anteriores, a fim de verificar as melhores intervenções para cada contexto. Nesse sentido, possui um alto nível de evidência e sua importância é significativa para a construção do conhecimento (Galvão; Ricarte, 2019). O procedimento sistemático ocorreu a partir das sete etapas detalhadas na sequência.

(1) Formulação da pergunta de pesquisa da revisão: nesta etapa, foi estabelecida a questão PICO, P = população (pacientes com câncer), I = intervenção (uso de REIKI associado à terapia convencional), C = controle/comparação (tratamento padrão para o câncer) e $\mathrm{O}=$ desfecho (melhora do bem-estar do paciente). Com isso, a interrogação feita foi "Em pacientes com câncer, o uso de Reiki associado ao tratamento convencional, em comparação apenas à terapêutica padrão promove a melhora do bem-estar e/ou quadro clínico?”.

(2) Busca na literatura: realizada em maio de 2021, com a utilização das bases de dados Biblioteca Virtual em Saúde (BVS) e Medical Publisher (PUBMED) para procurar estudos acerca da pergunta em questão.

(3) Seleção dos artigos: utilizaram-se os Descritores em Ciências da Saúde (DeCS) na combinação a partir do operador booleano AND: "Therapeutic Touch" AND Neoplasms and Therapeutics AND "Treatment Outcome" em todos os canais de busca. Incluíram-se os ensaios clínicos randomizados, com texto completo disponível. Foram excluídos os artigos que não tinham relação direta com o tema, associavam a outras terapias alternativas ou estavam repetidos em mais de uma base, permanecendo apenas uma vez. O processo de filtragem utilizou a Recomendação PRISMA ou Preferred Reporting Items for Systematic Reviews and Meta-Analyses (Moher et al., 2010).

Figura 1: Processo de pesquisa e escolha de estudos conforme recomendação PRISMA.

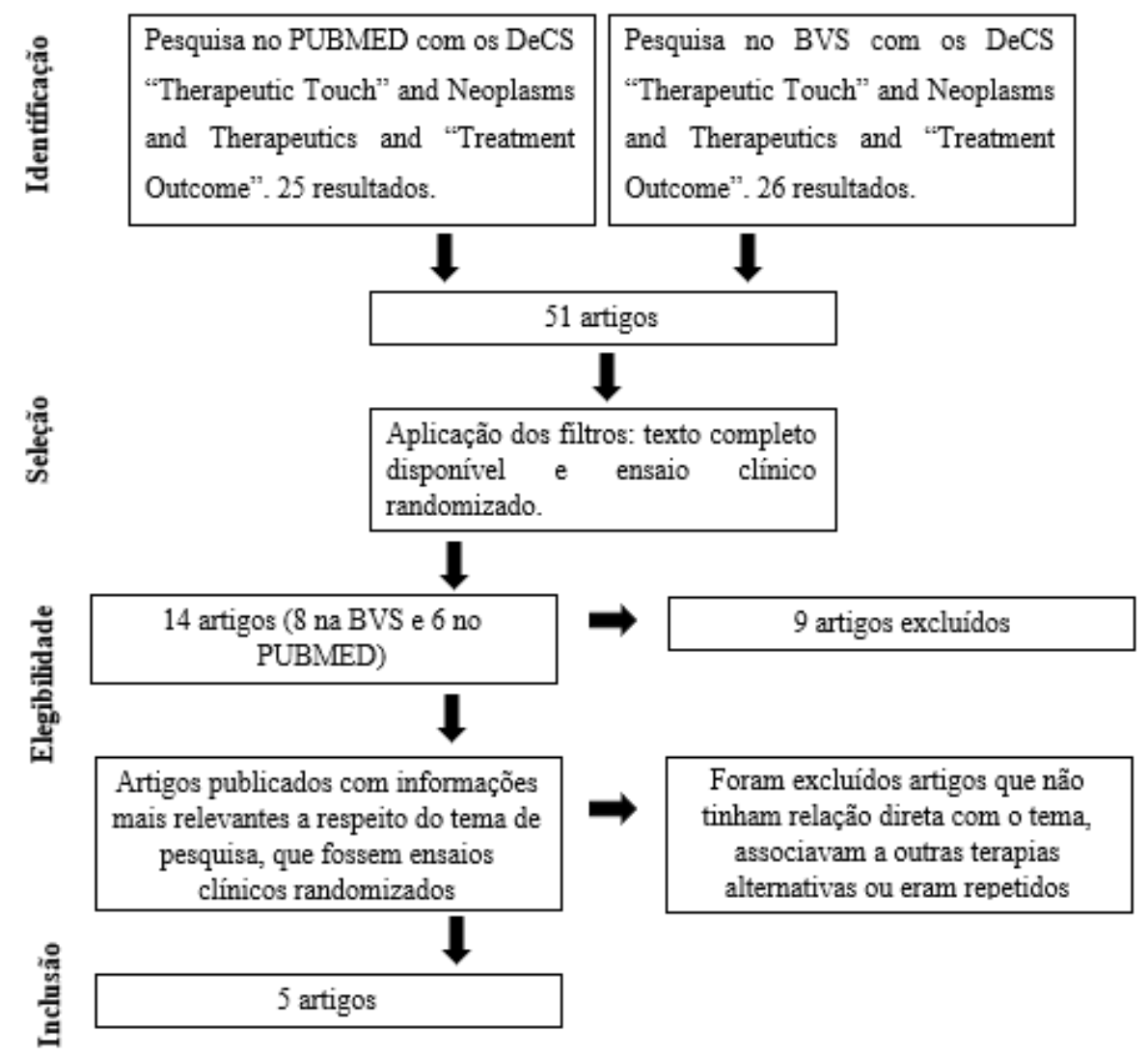

Fonte: Autores (2021). 
(4) Extração dos dados: buscou-se informações acerca da eficácia clínica do uso de Reiki associado quando comparado ao tratamento padrão isolado. Partindo disso, os dados foram usados na construção de quadros, que foram tidos como base para este trabalho.

(5) Avaliação da qualidade metodológica: a qualidade metodológica dos estudos da amostra final deste artigo foi classificada conforme as Diretrizes Metodológicas do Sistema GRADE (Brasil, 2014). A pirâmide de evidências utilizada para tal classificação foi a Classificação de Oxford Centre for Evidence-Based Medicine (Galvão et al., 2015), segundo a atualização de maio de 2001.

(6) Síntese de dados: foram selecionadas variáveis como: país de publicação, base de dados, tipo de estudo, nível de evidência, objetivo principal e principais resultados. Com isso, sintetizou-se as principais informações encontradas, especialmente no Quadro 2 foram feitas comparações dos resultados encontrados, a fim de possibilitar uma melhor análise dos estudos e encontrar uma resposta para a questão de pesquisa.

(7) Avaliação da qualidade das evidências: Detalhadamente, no quadro 3, foi empregado o sistema GRADE, que fornece a informação clara e concisa sobre a qualidade das evidências e o impacto das recomendações. Os estudos observacionais devem ser iniciados pela classificação mais baixa até a mais alta qualidade, de acordo com o documento.

Conforme esse sistema, os estudos podem ter nível de evidência: alto (forte confiança), moderado (moderada confiança), baixo (confiança imitada) ou muito baixo (pouca confiança). Isso se baseia em critérios como limitações metodológicas, inconsistências, evidências indiretas e imprecisão. Tais fatores, quando presentes, reduzem a confiabilidade da pesquisa.

\section{Resultados}

Conforme os dados do Quadro 1, evidencia-se que todos os estudos selecionados ( $\mathrm{N}=5)$ caracterizam-se como ensaios clínicos randomizados, com nível de evidência 1B. Além disso, aborda o objetivo principal de cada trabalho, direcionando o olhar para cada um deles.

Com relação aos países de publicação, todos foram publicados nos Estados Unidos, demonstrando uma centralização das pesquisas e a necessidade de expandi-las para as outras regiões do mundo, dada a importância do tema. Quanto às bases de dados, a maioria dos estudos $(\mathrm{N}=4)$ foi encontrada na BVS. 
Quadro 1: Caracterização geral dos artigos selecionados para compor a RS. Patos, 2021.

\begin{tabular}{|c|c|c|c|c|c|}
\hline Autores (Ano) & País & $\begin{array}{c}\text { Base de } \\
\text { dados }\end{array}$ & $\begin{array}{l}\text { Tipo de } \\
\text { estudo }\end{array}$ & $\begin{array}{c}\text { Nível de } \\
\text { evidência }\end{array}$ & Objetivo principal \\
\hline $\begin{array}{l}\text { Beard et al. } \\
(2011)\end{array}$ & $\begin{array}{l}\text { Estados } \\
\text { Unidos }\end{array}$ & BVS & ECR & 1B & $\begin{array}{l}\text { Examinar os efeitos clínicos de } 2 \text { terapias } \\
\text { complementares (CAM), terapia de } \\
\text { resposta de relaxamento (RRT) e terapia } \\
\text { de Reiki, em homens sendo tratados com } \\
\text { radioterapia por feixe externo (EBRx) } \\
\text { para câncer de próstata. }\end{array}$ \\
\hline $\begin{array}{l}\text { Fitzhenry et al. } \\
\text { (2013) }\end{array}$ & $\begin{array}{l}\text { Estados } \\
\text { Unidos }\end{array}$ & BVS & ECR & $1 \mathrm{~B}$ & $\begin{array}{l}\text { Investigar o efeito do toque curativo (HT) } \\
\text { na fadiga em pacientes com câncer de } \\
\text { mama submetidas à radioterapia (RT). }\end{array}$ \\
\hline $\begin{array}{l}\text { Hart et al. } \\
(2011)\end{array}$ & $\begin{array}{l}\text { Estados } \\
\text { Unidos }\end{array}$ & BVS & ECR & $1 \mathrm{~B}$ & $\begin{array}{l}\text { Descrever o uso integrativo do toque de } \\
\text { cura da terapia de biofield em conjunto } \\
\text { com a quimiorradiação recebida por } \\
\text { pacientes com câncer cervical (estágios } \\
\text { IB1 a IVA), conforme relatado em um } \\
\text { estudo de pesquisa de } 2010 \text {. }\end{array}$ \\
\hline $\begin{array}{l}\text { Lutgendorf et } \\
\text { al. (2010) }\end{array}$ & $\begin{array}{l}\text { Estados } \\
\text { Unidos }\end{array}$ & PUBMED & ECR & $1 \mathrm{~B}$ & $\begin{array}{l}\text { Examinar os efeitos de uma terapia } \\
\text { complementar, Healing Touch (HT), } \\
\text { versus treinamento de relaxamento (RT) e } \\
\text { cuidados habituais (UC) para (1) apoiar a } \\
\text { imunidade celular, (2) melhorar o humor } \\
\text { e a qualidade de vida (QV), e (3) redução } \\
\text { das toxicidades associadas ao tratamento } \\
\text { e atraso no tratamento em pacientes com } \\
\text { câncer cervical que recebem } \\
\text { quimiorradiação. }\end{array}$ \\
\hline $\begin{array}{l}\text { Wong et al. } \\
\text { (2012) }\end{array}$ & $\begin{array}{l}\text { Estados } \\
\text { Unidos }\end{array}$ & BVS & ECR & 1B & $\begin{array}{l}\text { Avaliar a viabilidade da administração de } \\
\text { TH em unidades de internação e } \\
\text { ambulatorial de oncologia pediátrica do } \\
\text { Kapi'olani Medical Center for Women } \\
\text { and Children. }\end{array}$ \\
\hline
\end{tabular}

Fonte: Dados de Pesquisa (2021).

No Quadro 2, foram expostos os principais resultados dos 5 estudos abordados na pesquisa. Nesse sentido, constatouse que um deles $(\mathrm{N}=1)$ diz não ter diferença significativa na qualidade de vida quando comparamos Reiki e tratamento padrão, além dos pacientes do Reiki relatarem mais fadiga. No entanto, outros dois $(\mathrm{N}=2)$ trazem informações que vão de encontro ao primeiro, como melhora de dor, estresse e fadiga, inclusive pela percepção dos pais sobre seus filhos, além de, ao final da intervenção, relatarem melhora na subescala de bem-estar emocional da escala de qualidade de vida e na ansiedade. Outrossim, os demais $(\mathrm{N}=2)$ evidenciam uma queda menor da citotoxicidade das células natural killer e uma redução do humor deprimido nos pacientes que receberam Reiki em relação aos que fizeram terapia de relaxamento ou tratamento padrão. 
Quadro2: Principais Resultados. Patos, 2021.

\begin{tabular}{|c|c|}
\hline $\begin{array}{l}\text { Autores } \\
\text { (Ano) }\end{array}$ & Principais Resultados \\
\hline $\begin{array}{l}\text { Beard et al. } \\
(2011)\end{array}$ & $\begin{array}{l}\text { Nenhuma diferença estatisticamente significativa foi encontrada entre os grupos Terapia de } \\
\text { Resposta de Relaxamento (RRT), Reiki e controle nos escores totais, em qualquer momento, dos } \\
\text { instrumentos The State - Trait Anxiety Inventory (STAI), Center for Epidemiological Scale - } \\
\text { Depression (CES-D) ou Functional Assessment of Cancer Therapy - General (FACT-G), as quais } \\
\text { avaliam, respectivamente, ansiedade, depressão e qualidade de vida. No entanto, no final da } \\
\text { intervenção, uma melhora significativa foi encontrada na subescala de bem-estar emocional da } \\
\text { escala de qualidade de vida do FACT-G no grupo RRT em comparação com os grupos Reiki e } \\
\text { controle (P }=0,01) \text {. Nos participantes que foram classificados como "ansiosos" no início do } \\
\text { estudo, uma melhora estatisticamente significativa ocorreu no grupo RRT (P =0,02), e uma } \\
\text { tendência positiva foi encontrada no grupo Reiki }(\mathrm{P}=0,10) \text {. }\end{array}$ \\
\hline $\begin{array}{l}\text { Fitzhenry et } \\
\text { al. (2013) }\end{array}$ & $\begin{array}{l}\text { Os participantes do Reiki tendiam a relatar níveis mais elevados de fadiga, estatisticamente } \\
\text { significativos para interferência }(=0,010) \text { e fadiga normal }(=0,024) \text {. O grupo controle tendeu a } \\
\text { relatar maiores reduções na fadiga em relação às suas próprias médias do que o grupo Healing } \\
\text { Touch (HT) ou Reiki (Cohen's }=0,30 \text { a } 0,49 \text { vs } 0,06 \text { a } 0,18 \text {, respectivamente). Não houve } \\
\text { diferenças estatisticamente significativas entre os grupos para qualidade de vida. }\end{array}$ \\
\hline $\begin{array}{l}\text { Hart et al. } \\
(2011)\end{array}$ & $\begin{array}{l}\text { Os resultados indicaram efeitos sobre a resposta imunológica e depressão em receptores de Reiki } \\
\text { em comparação com pacientes que recebem relaxamento ou tratamento padrão. Especificamente, } \\
\text { os receptores de Reiki demonstraram uma queda mínima de citotoxicidade das células natural } \\
\text { killers ao longo do tratamento, enquanto a citotoxicidade dos pacientes recebendo terapia de } \\
\text { relaxamento e tratamento padrão diminuiu drasticamente durante a radiação. Os destinatários do } \\
\text { Reiki também mostraram diminuição no humor deprimido em comparação com a terapia de } \\
\text { relaxamento e os destinatários de tratamento padrão. }\end{array}$ \\
\hline $\begin{array}{l}\text { Lutgendorf } \\
\text { et al. (2010) }\end{array}$ & $\begin{array}{l}\text { Os pacientes com Reiki tiveram uma diminuição mínima na citotoxicidade das células natural } \\
\text { killers }(\mathrm{NK}) \text { ao longo do tratamento, enquanto os pacientes com terapia de relaxamento (RT) e } \\
\text { tratamento padrão dimucinuíram acentuadamente durante a quimiorradiação (grupo por interação } \\
\text { de tempo: p =0,018). Pacientes com Reiki mostraram maiores reduções em dois indicadores } \\
\text { diferentes de humor deprimido (subescala de humor deprimido CES-D e escala de depressão } \\
\text { POMS) em comparação com RT e tratamento padrão (grupo por interações de tempo: p <0,05). } \\
\text { Nenhuma diferença entre os grupos foi observada na qualidade de vida, atraso no tratamento ou } \\
\text { toxicidades avaliadas clinicamente. }\end{array}$ \\
\hline $\begin{array}{l}\text { Wong et al. } \\
(2012)\end{array}$ & $\begin{array}{l}\text { Aqueles no grupo Reiki mostraram diminuições significativas nas pontuações de dor, estresse e } \\
\text { fadiga para os participantes, pais e cuidadores. Além disso, a percepção dos pais sobre a dor de } \\
\text { seus filhos diminuiu significativamente para o grupo Reiki em comparação com o grupo que } \\
\text { recebeu atividades de leitura / brincadeira. }\end{array}$ \\
\hline
\end{tabular}

Fonte: Dados de Pesquisa (2021).

A qualidade das evidências, apresentadas no Quadro 3, foi classificada observando a existência ou ausência de fatores que aumentam e diminuem a qualidade destas evidências, em conformidade com o sistema GRADE em alto. Considerando que os aspectos que decrescem o nível de uma evidência no ECR (limitações metodológicas, inconsistência, evidência indireta, imprecisão) não foram identificados, e todos os estudos ( $\mathrm{N}=5)$ obtiveram qualidade alta.

Quadro 3: Avaliação da qualidade dos estudos selecionados nessa pesquisa.

\begin{tabular}{|l|c|c|c|c|c|}
\hline Autores (Ano) & $\begin{array}{c}\text { Limitações } \\
\text { Metodológicas }\end{array}$ & Inconsistência & $\begin{array}{c}\text { Evidência } \\
\text { indireta }\end{array}$ & Imprecisão & Qualidade \\
\hline Beard et $a l$. (2011) & Não há & Não há & Não há & Não há & Alta \\
\hline $\begin{array}{l}\text { Fitzhenry } \text { et } a l . \\
(2013)\end{array}$ & Não há & Não há & Não há & Alta \\
\hline Hart et al. $(2011)$ & Não há & Não há & Não há & Não há & Alta \\
\hline $\begin{array}{l}\text { Lutgendorf } \text { et } a l . \\
(2010)\end{array}$ & Não há & Não há & Não há & Não há & Alta \\
\hline Wong et al. $(2012)$ & Não há & Não há & Não há & Não há & Alta \\
\hline
\end{tabular}

Fonte: Dados de Pesquisa (2021). 


\section{Discussão}

As Terapias Integrativas Complementares são práticas terapêuticas que tratamo indivíduo de forma individual e holística, buscando encontrar o equilíbrio de forma autoeducativa e não alopática. Dentre essas terapias, encontra-se o Reiki, cuja palavra tem origem japonesa, onde rei significa universal e $k i$ energiavital, denominando, então, "energia vital universal" (Corrêa et al., 2020). Terapias como esta prestam assistência à integralidade do homem doente, fomentada pela empatia, o respeito e a autonomia (Locateli et al., 2020).

A terapia Reiki, como outras terapias de bioenergia, está enraizada em conceitos de compaixão, intenção positiva, autocapacitação, mente-corpo tríade espiritual e tendência inata do corpo para a cura (Wong et al., 2012). Reiki é um tipo de terapia energética usada para melhorar a capacidade do corpo de se curar. Um número limitado de estudos de pesquisa cientificamente rigorosos explorou a eficácia do Reiki e um deles sugeriu efeitos benéficos na dor, fadiga, humor e qualidade de vida (Beard et al., 2011).

O uso de terapia Reiki em pacientes com câncer cervical submetidos a tratamento quimioterápico demonstrou redução mínima da atividade das células natural killer, em comparação ao tratamento padrão, que apresenta declínios significativos (Hart et al., 2011). É importante lembrar que as células natural killer (NK) são de extrema importância na vigilância imunológica para metástases, pois o sistema imunológico possui mecanismos de defesa contra a célula tumoral capazes de lisar e induzir apoptose das células cancerosas (Duarte; Barros, 2016).

A preservação do efeito citotóxico dessas células é muito importante, já que pode significar uma progressão mais lenta da neoplasia. Observou-se redução do humor deprimido nos pacientes com câncer cervical tratados com quimioterapia e Reiki, enquanto os que fizeram apenas tratamento padrão permaneceram com depressão clínica. As técnicas usadas na pesquisa de câncer cervical foram conexão de chakra, drenagem do fígado, limpeza magnética, limpeza da mente e preparação do praticante (Hart et al., 2011).

Existe o risco de substituição do tratamento convencional pelo Reiki, por isso é importante lembrar que este é uma terapia alternativa, cujas técnicas e preparação do profissional devem ser qualificadas a fim de proporcionar melhores resultados. Se aplicado isoladamente, o Reiki pode causar progressão rápida da doença, indo em sentido contrário ao processo de cura. Portanto, ao apoiar esses recursos em consonância com as terapias convencionais, o resultado pode ser aprimorado e o paciente beneficiado (Hart et al., 2011).

Um estudo piloto realizado com mulheres diagnosticadas com câncer de mama e submetidas à radioterapia avaliou o impacto do Reiki no sintoma da fadiga generalizada e da qualidade de vida decorrentes das terapias convencional e cirúrgica. Neste, um grupo de pessoas recebeu o tratamento Reiki, enquanto outro recebeu apenas placebo. Apesar de a hipótese basear-se na melhora da fadiga durante o tratamento, e o aprimoramento da qualidade de vida ao término da terapia, tal pesquisa revelou que os pacientes apresentaram níveis maiores de fadiga, e não houve nenhuma implicação significativa na qualidade de vida, assim o efeito hipotético não foi sustentado (Fitzhenry et al., 2013). Os autores enfatizam que, no entanto, que fatores como o descanso e a empatia do indivíduo são elementos que podem influenciar na resposta terapêutica, portanto tornando-se fundamental ao estudo das condições para futuras pesquisas.

Em pacientes com câncer cervical submetidas a quimioterapia, o Reiki aumentou a citotoxicidade das células natural killer quando comparado aos grupos de controle. Há algumas evidências de que a terapia pode aumentar a energia em pacientes em tratamento para o câncer (Hart et al., 2011).

Outro estudo examinou os efeitos do Reiki ou terapia de resposta ao relaxamento (TRS) com reestruturação cognitiva em 18 pacientes com câncer de próstata submetidos à radioterapia externa (Beard et al., 2011). Os autores, ao final da intervenção, constaram que mais da metade dos pacientes relataram redução da ansiedade, destacando essa tendência positiva no balanço dos níveis ansiosos. 
Contudo, sua análise estatística não identificou nenhuma diferença com relação aos resultados psicossociais baseados nas medidas Spielberger State Anxiety Inventory (STAI), Center for Epidemiologic Studies Depression (CES) e Functional Assessment of Cancer Therapy-General (FACT-G), como também não demonstrou melhorias no sentido do bem-estar emocional. Destaca-se o tamanho relativamente pequeno da amostra analisada como limitação do estudo, de modo a não estender para populações mais heterogêneas, pois abre margem a demais interpretações dissonantes com a realidade (Beard et al., 2011).

Sabe-se que o diagnóstico de uma doença com risco de morte em crianças é um evento profundamente estressante que pode afetar o bem-estar físico, emocional, mental e espiritual de toda a família. Embora grandes avanços tenham sido feitos no tratamento de doenças malignas na infância, as complicações das terapias são significativas. As crianças costumam sentir dor, ansiedade, estresse e fadiga como resultado da quimioterapia e de outras modalidades de tratamento usadas na terapia do câncer. Pesquisas recentes sugerem que terapias alternativas ou complementares são eficazes na redução dos efeitos colaterais da quimioterapia ou radioterapia (Wonget al., 2012).

A Healing Touch (HT) ou Reiki, terapia complementar não invasiva de biocampo, demonstrou eficácia na redução da dor, angústia e fadiga em pacientes adultos com câncer recebendo quimioterapia e/ou radioterapia. Supostamente apóia o processo natural de cura do corpo e aumenta a função do sistema imunológico. Praticantes treinados usam suas mãos para manipular a energia por meio de toques leves ou mãos sobre o corpo para equilibrar, limpar e energizar o sistema de energia humano. Esta manipulação de energia apóia e facilita a saúde física, emocional, mental e espiritual do indivíduo submetido ao toque terapêutico (Wong et al., 2012).

A terapia Reiki, em geral, é dividida em quatro fases: a centralização da consciência, a avaliação do campo de energia do paciente, o reequilíbrio ou repadronização da energia e a reavaliação do campo de energia do paciente (Marta et al., 2010). Tem sido usada em muitos hospitais e centros médicos acadêmicos em todo o Havaí, conforme estudo analisado. Ela é oferecida para pacientes oncológicos no Kapi'olani Medical Center for Women and Children (KMCWC) desde 1999. Embora tenha se mostrado eficaz na população adulta, existem pesquisas limitadas sobre a eficácia da terapia na população pediátrica (Wong et al., 2012.)

Em outra análise, foram examinados os efeitos da terapia complementar, a terapia Reiki, versus treinamento de relaxamento (RT) e cuidado usual (UC) em pacientes com câncer cervical que receberam quimiorradiação. A HT demonstrou efeitos positivos na preservação da citotoxicidade de células NK ao longo do curso do tratamento, enquanto células NK de pacientes com RT e UC diminuíram acentuadamente durante a quimiorradiação (Lutgendorf et al., 2010).

Pacientes em uso da HT mostraram maiores reduções em dois indicadores diferentes de humor deprimido em comparação com treinamento de relaxamento e cuidado usual. Nenhum efeito da HT foi observado para a qualidade de vida, atraso no tratamento ou toxicidades classificadas clinicamente. Percebe-se, por conseguinte, que a terapia em questão pode beneficiar pacientes com câncer cervical ao moderar os efeitos da quimiorradiação no humor deprimido e na imunidade celular (Lutgendorf et al., 2010).

Ao considerar o Reiki como estratégia de cuidado no contexto da depressão, propicia-se o fortalecimento dos preceitos de humanização no atendimento de profissionais de saúde, por meio do acolhimento, da escuta qualificada, da inclusão social e integralidade, viabilizando ações capazes de promoverem uma resposta adequada e efetiva à crescente demanda das pessoas acometidas por essa psicopatologia (Medeiros et al., 2020).

\section{Considerações Finais}

Considerando os estudos sobre Reiki associado as terapêuticas oncológicas padrão, concluiu-se que a terapia de toque é um tratamento alternativo que propicia melhora dos sintomas como diminuição da dor, relaxamento, redução de estresse, ansiedade, fadiga, como também aumentou a vivacidade e o humor dos pacientes, e em alguns casos houve uma redução de 
células natural killers, contendo relação com a citotixicidade dessas e aumento significativo na hemoglobina e no hematócrito de algumas pacientes. O uso do Reiki no período das quimioterapias, foi uma intervenção que limitou os efeitos adversos desse tratamento clássico nos pacientes com câncer de diferentes idades.

Portanto, essa terapia alternativa pode ser um instrumento favorável no gerenciamento dos pacientes oncológicos, visto que diminui os problemas emocionais dos pacientes, atendendo suas necessidades emocionais e aumentando a qualidade de vida durante o recurso terapêutico padrão.

Recomenda-se para trabalhos futuros, em função do número pequeno de pessoas que receberam a terapia Reiki na maioria dos estudos analisados, aumentar a quantidade de grupos que receberão a terapia em questão, além da necessidade de apontar mais claramente as evidências dos estudos para uma maior confiabilidade dos dados. Mesmo com forte grau de evidência dos estudos e ainda que o presente estudo possa adicionar na prática complementar em pacientes diagnosticados com câncer, ainda é preciso acrescer as pesquisas cientificas sobre o tema, em virtude desse tema ser importante na qualidade de vida dos pacientes com câncer e humanização do tratamento desses, pelos profissionais da saúde.

\section{Referências}

Alarcão, Z. \& Fonseca, J. R. S. (2016). The effect of Reiki therapy on quality of life of patients with blood cancer: results from a randomized controlled trial. European Journal Of Integrative Medicine. 8(3), 239-49. http://dx.doi.org/10.1016/j.eujim.2015.12.003.

Amarello, M. M., Catellanos, M. E. P. \& Souza, K. M. J. (2021). Reiki therapy in the Unified Health System: meanings and experiences in integral health care. Revista Brasileira de Enfermagem. 74(1), 1-7. http://dx.doi.org/10.1590/0034-7167-2019-0816.

Batista, D. R. R., Mattos, M. \& Silva, S. F. (2015). Convivendo com o câncer: do diagnóstico ao tratamento. Revista de Enfermagem da UFSM. 5(3), 499-51. http://dx.doi.org/10.5902/2179769215709.

Beard, C., Stason, W., Wang, Q., Manola, J., Dean-Clower, E., \& Dusek, J. et al. (2010). Effects of complementary therapies on clinical outcomes in patients being treated with radiation therapy for prostate cancer. Cancer, 117(1), 96-102. 10.1002/cncr.25291.

Brasil. Ministério da Saúde. Secretaria de Atenção à Saúde. Departamento de Atenção Básica. (2015). Política nacional de práticas integrativas e complementares no SUS: atitude de ampliação de acesso (2), 1-98. Brasília: Ministério da Saúde.

Brasil. Ministério da Saúde. Secretaria de Ciência, Tecnologia e Insumos Estratégicos. Departamento de Ciência e Tecnologia. (2014). Diretrizes metodológicas: Sistema GRADE - Manual de graduação da qualidade da evidência e força de recomendação para tomada de decisão em saúde (1),1-74. Brasília: Ministério da Saúde.

Corrêa, E. A. B., Araújo, A. H. I. M. de, \& Mendes, M. I. de O. I. (2020). A terapia reiki e outras terapias integrativas complementares no tratamento de enfermidades. Revista JRG De Estudos Acadêmicos, 3(7), 561-576.

DUARTE, Mariane Queiroz. Ação das células imunitárias natural killer contra células tumorais. 2016. 23 f. Monografia (Graduação). Disponível a partir de https://repositorio.uniceub.br/jspui/handle/235/11074.

FitzHenry, F., Wells, N., Slater, V., Dietrich, M., Wisawatapnimit, P., \& Chakravarthy, A. (2013). A Randomized Placebo-Controlled Pilot Study of the Impact of Healing Touch on Fatigue in Breast Cancer Patients Undergoing Radiation Therapy. Integrative Cancer Therapies, 13(2), 105-113. $10.1177 / 1534735413503545$.

Galvão, M., \& Ricarte, I. (2019). Revisão Sistemática Da Literatura: Conceituação, Produção E Publicação. Logeion: Filosofia Da Informação, 6(1), 57-73. 10.21728/logeion.2019v6n1.p57-73.

Galvão, T. F., Pansani, T. S. A., Harrad, D. Principais itens para relatar Revisões sistemáticas e Meta-análises: A recomendação PRISMA. (2015). Epidemiologia E Serviços De Saúde, 24(2), 335-342. 10.5123/s1679-49742015000200017.

Hart, L., Freel, M., Haylock, P., \& Lutgendorf, S. (2011). The Use of Healing Touch in Integrative Oncology. Clinical Journal Of Oncology Nursing, 15(5), 519-525. 10.1188/11.cjon.519-525.

Kirshbaum, M., Stead, M., \& Bartys, S. (2016). An exploratory study of reiki experiences in women who have cancer. International Journal Of Palliative Nursing, 22(4), 166-172. 10.12968/ijpn.2016.22.4.166.

Locateli, G., Chagas, N., Menzel Gato, C., Gonçalves de Oliveira, G., \& Terezinha Zenevicz, L. (2020). Acendendo as Luzes: uma inovação no Cuidado a Saúde dos Pacientes Oncológicos, Familiares e Equipe. Saúde Em Redes, 6(1), 155-162.

Lutgendorf, S., Mullen-Houser, E., Russell, D., DeGeest, K., Jacobson, G., \& Hart, L. et al. (2010). Preservation of immune function in cervical cancer patients during chemoradiation using a novel integrative approach. Brain, Behavior, And Immunity, 24(8), 1231-1240. 10.1016/j.bbi.2010.06.014.

Marta, I., Baldan, S., Berton, A., Pavam, M., \& Silva, M. (2010). Efetividade do Toque Terapêutico sobre a dor, depressão e sono em pacientes com dor crônica: ensaio clínico. Revista Da Escola De Enfermagem Da USP, 44(4), 1100-1106. 10.1590/s0080-62342010000400035. 
Research, Society and Development, v. 10, n. 9, e10210917751, 2021

(CC BY 4.0) | ISSN 2525-3409 | DOI: http://dx.doi.org/10.33448/rsd-v10i9.17751

Medeiros, S., Oliveira, A., Silva, M., Freitag, V., Afonso, M., \& Brum, A. (2020). Práticas integrativas e complementares: estratégia de cuidado por meio do Reiki em pessoas com depressão. Research, Society And Development, 9(2), e127922149. 10.33448/rsd-v9i2.2149.

Moher, D., Liberati, A., Tetzlaff, J., \& Altman, D. (2010). Preferred reporting items for systematic reviews and meta-analyses: The PRISMA statement. International Journal Of Surgery, 8(5), 336-341. 10.1016/j.ijsu.2010.02.007.

Nascimento, J., Sousa, M., \& Alencar, T. (2020). Escalas sobre qualidade de vida em pacientes com câncer de mama / Quality of life scales in breast cancer patients. Brazilian Journal Of Health Review, 3(6), 16559-16578. 10.34119/bjhrv3n6-077.

Wong, J., Ghiasuddin, A., Kimata, C., Patelesio, B., \& Siu, A. (2012). The Impact of Healing Touch on Pediatric Oncology Patients. Integrative Cancer Therapies, 12(1), 25-30. 10.1177/1534735412446864. 